\title{
Study on Corrosion Inhibition and Adsorption of Polyaspartic Acid Corrosion Inhibitor to Seawater Copper
}

\author{
Lihua Liang, Changjiang Zuo, Xin Shi \\ Qingdao technical college, Qingdao, Shandong, China
}

Keywords: copper; sea water; PASP; adsorption and corrosion

\begin{abstract}
The inhibitory performance and adsorption behavior of green inhibitor polyaspartic acid (PASP) on copper in 3\% $\mathrm{NaCl}$ solution was studied by electrochemical impedance and polarization curve method. The results showed that when PASP was used at an optimal concentration of 15 $\mathrm{mg} / \mathrm{L}$, the corrosion inhibition rate could reach $77.2 \%$. The adsorption of PASP significantly reduced the erosion of $\mathrm{Cl}-$, which belongs to the anode type corrosion inhibitor. The adsorption behavior of PASP obeys the Langmuir adsorption isotherm. The formula is a spontaneous and exothermic process that belongs to chemical adsorption.
\end{abstract}

\section{Introduction}

As the coastal areas are facing a crisis of severe shortage of fresh water resources, it is imperative to vigorously develop and use sea water resources [1-3]. As an industrial cooling water, seawater not only helps alleviate the problems of the shortage of fresh water that is inconsistent with industrial development, but also has the advantages of low water intake temperature, good cooling effect, and simple operation and management. Since the seawater circulation cooling technology has a small amount of water and a low water price, it is economically more reasonable. In the circulating water cooling system material, the application of steel is more common, but relative to copper, the steel is more susceptible to corrosion in seawater [4]. Copper has become an important industrial material due to its high positive potential, excellent heat conduction and mechanical processing properties, and excellent corrosion resistance and other characteristics. It has begun to replace carbon steel in electronic equipment in more fields. However, copper is also susceptible to different forms of corrosion through different corrosive factors, such as the corrosion promoting effects of chlorides, sulfates, nitrates, etc., particularly in chloride-ion containing media $[5,6]$.

For corrosion of metals, the use of corrosion inhibitor is a cost-effective method. Copper corrosion inhibitors are required for pickling. Most of the acid and corrosion inhibitors contain nitrogen and sulfur. Oxygen-containing organic compounds can form a corrosion-inhibiting film on the surface of copper to reduce corrosion. With the increasing awareness of environmental protection for humans, environmentally-friendly corrosion inhibitors become the development direction of copper corrosion inhibitors as corrosion inhibitors in industrial cooling water treatment chemicals. In the 1970s, the application of corrosion inhibitors of $\mathrm{Cr}$ series and P series was relatively popular, but in recent years it has been limited by the environment [7].

At present, the development of environmentally friendly corrosion inhibitors that are non-toxic and conducive to environmental protection has been developed industrially. Polyaspartic acid (abbreviated as PASP) is a biopolymer developed in recent years and has excellent scale inhibition and dispersion properties and good biodegradability. In neutral systems, the study of polyaspartic acid as a metal corrosion inhibitor has become a hot topic at home and abroad. Especially in the acidic environment, polyaspartic acid is a green product [8, 9]. It is a polymer made from the shrinkage of amino and carboxyl groups of aspartic acid monomers, and can be completely biodegraded into substances harmless to the environment. It is non-toxic. Non-pollution, is a kind of environment-friendly polymer research found that polyaspartic acid has various functions such as corrosion inhibition, scale inhibition, chelation, moisturizing, and dispersion, and the economic and 
social benefits are very considerable.

In this paper, the corrosion inhibition and adsorption behavior of polyaspartic acid (PASP) for copper in seawater was studied by electrochemical method.

\section{Corrosion Mechanism of Copper in Seawater}

\subsection{Electrochemical Characteristics of Corrosion in Seawater and Simulated Seawater}

Seawater is a typical electrolyte, so the basic laws of electrochemical corrosion are applicable to seawater corrosion [10]. However, starting from the characteristics of seawater, the electrochemical process of seawater corrosion has the following features:

1) Halogen ions such as chlorine ions in seawater can hinder and destroy the passivation of metals, and the anodic process of seawater corrosion is easier to perform. The destructive effects of chloride ions including: osmotic destruction of oxide films and destructive destruction of colloidal protective films; easier adsorption than certain deactivators; adsorption on metal surfaces or on thin passive films, formating strong electric field which makes the metal ions easy to elute; the complex with metal to chlorine accelerates the dissolution of the metal. All these effects can reduce the anodic polarization retardation and cause the seawater to be highly corrosive to metals.

2) The cathode depolarizer for seawater corrosion is oxygen, and the cathode process is the controlling link of the corrosion reaction. At the $\mathrm{pH}$ value of seawater, the corrosion of most metals in seawater depends on oxygen depolarization.

3) The resistive block of seawater corrosion is small, and the contact of dissimilar metals can cause significant galvanic corrosion.

4) In seawater, local corrosion such as pitting corrosion and crevice corrosion is prone to occur due to the. local destruction of passivation.

\subsection{Corrosion mechanism of copper}

The content of oxygen and Cl- in the chloride medium is the main factor affecting the corrosion. $\mathrm{Cl}$ - can increase the corrosion activity, damage the passivation film on the metal surface, and easily generate pitting corrosion.

When the mass concentration of $\mathrm{Cl}$ - is less than $1 \mathrm{~mol} / \mathrm{L}$, the reactions shown in Figure 1 occur when the copper is corroded:

$$
\begin{array}{ll}
\text { Anode: } & \\
& \mathrm{Cu}+\mathrm{Cl}^{-} \longrightarrow \mathrm{CuCl}+\mathrm{e} \\
& \mathrm{CuCl}+\mathrm{Cl}^{-} \longrightarrow \mathrm{CuCl}+\mathrm{e} \\
\text { Cathode } & \longrightarrow \\
& \mathrm{O}_{2}+2 \mathrm{H}_{2} \mathrm{O}+4 \mathrm{e} \longrightarrow \mathrm{OH}^{-}
\end{array}
$$

Fig.1 Electrode reaction when copper is corroded

The use of corrosion inhibitors is one of the important means to control the corrosion of copper and copper alloys.

\section{Experimental Materials and Methods}

\subsection{Electrochemical test system}

The experimental material is pure copper (>99.9\%). The pure copper is cut into electrodes(10 $\mathrm{mm} \times 5 \mathrm{~mm} \times 1 \mathrm{~mm}$ ) and the epoxy resin is solid-sealed on its non-working surface, get a working surface area of $0.5 \mathrm{~cm} 2$. Before test, the electrode is polished by metallographic sandpaper, then washed by ionized water, anhydrous ethanol, and deionized water and dried with cold air. When using electrochemical calorimetry, use a saturated calomel electrode as the reference electrode and a Pt electrode as the counter electrode. The etching solution used is a $3 \% \mathrm{NaCl}$ solution (analytically 
pure) made of deionized water. Corrosion inhibitor PASP is homemade with a molecular weight of 5000. The experimental temperature is controlled by the constant temperature water bath.

\subsection{Test method}

The electrode was soaked in the solution for $1 \mathrm{~h}$ and tested for AC impedance and polarization curves. The frequency range of the AC impedance was $100 \mathrm{kHz}-0.05 \mathrm{~Hz}$. The equivalent circuit was analyzed and processed by EQUIVCRT software.

\section{Results and Discussion}

\subsection{Polarization curve}

The pure copper electrode was immersed in $3 \% \mathrm{NaCl}$ simulated sea solutions with different PASP concentarions for 30 minutes to perform polarization curve test. Figure 2 shows the polarization curve obtained by the test. As can be seen from Figure 2 , the self-corrosion potentials of the different citric acid solutions are positively shifted compared to the citric acid stock solutions. After adding different corrosion inhibitors, it can be seen from the figure that both the anode and the anode reactions been somewhat inhibited. It shows that the addition of corrosion inhibitors(PASP) well inhibits the corrosion of pure copper electrodes.

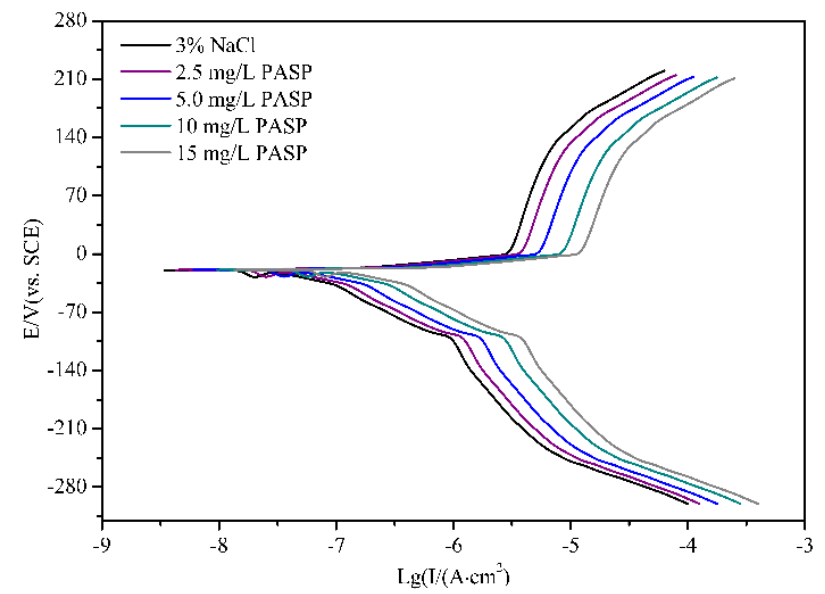

Fig.2 Polanization curves for copper in sea water in the different concentrations of PASP

The corrosion inhibition efficiency is calculated according to the following formula:

$$
\eta=\frac{J_{0}-J_{\text {corr }}}{J_{0}} \times 100 \%
$$

In the formula, $\mathrm{J}_{0}$ represents the self-corrosion current density of the pure copper electrode in the citric acid stock solution; Jcorr represents the self-corrosion current density of the pure copper electrode after adding different corrosion inhibitors.

The corresponding electrochemical parameters are shown in Table 1. As can be seen from Table 1 , under the same conditions, add more PASP would get a better effect.

Table 1 Elaetrochemical parameters of copper electrodes with different concentrations of PASP at $25^{\circ} \mathrm{C}$

\begin{tabular}{|c|c|c|c|c|c|}
\hline $\begin{array}{c}\mathrm{C}_{\text {PASP }} \\
(\mathrm{mg} / \mathrm{L})\end{array}$ & $\begin{array}{c}\text { Icorr } \\
\left(\mathrm{uA} / \mathrm{cm}^{2}\right)\end{array}$ & Ecorr/mV(vs SCE $)$ & $\beta_{a}(\mathrm{mv})$ & $\beta_{c}(\mathrm{mv})$ & $\eta(\%)$ \\
\hline 0 & 3.266 & -56.8 & 110.6 & 137.3 & - \\
\hline 2.5 & 1.652 & -54.2 & 188.5 & 125.6 & 49.4 \\
\hline 5 & 0.986 & -51.3 & 436.5 & 201.4 & 69.8 \\
\hline 10 & 0.865 & -48.2 & 556.7 & 235.4 & 73.5 \\
\hline 15 & 0.745 & -44.7 & 671.5 & 243.7 & 77.2 \\
\hline
\end{tabular}


$\beta_{a}$ :anodic Tafel slop; $\beta_{c}$ :cathodic Tafel slope; $\eta$ : inhibition efficiency; $\mathrm{E}_{\text {corr }}$ : corrosion potential

With the addition of PASP, the corrosion current density of the electrode gradually decreases. When the PASP concentration is $15 \mathrm{mg} / \mathrm{L}$, the corrosion current density is the smallest in the $3 \%$ $\mathrm{NaCl}$ simulated sea water corrosion solution. The amount of PASP added is $15 \mathrm{mg} / \mathrm{L}$ is the best, and the corrosion inhibition rate can reach $77.2 \%$. The tendency of Tafel slope in the anode area is greater than the Tafel rate in the cathode area, which can be judged by the corrosion potential. So the PASP is an anode typ corrosion inhibitor under this condition.

\subsection{Impedance spectrum}

The impedance spectrums of copper electrode when treated with different concentrations of PASP were tested using the simulated analog circuit shown in Figure 3.

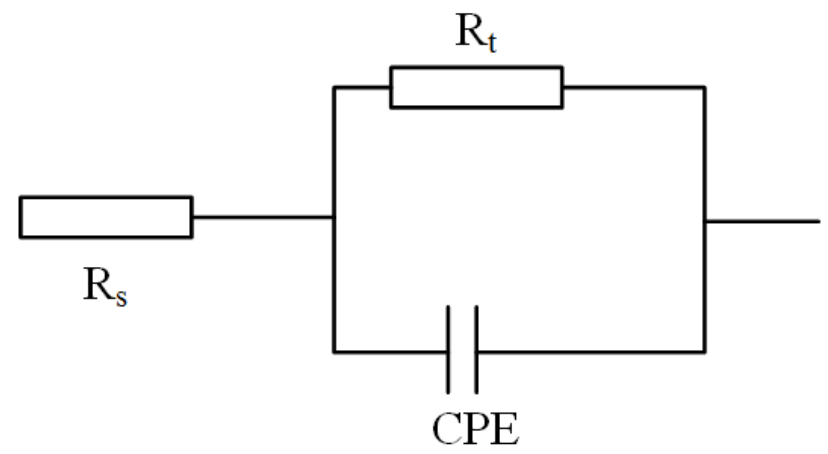

Fig.3 Power power short-run balance

$\mathrm{R}_{\mathrm{s}}$ is the solution resistance, $\mathrm{R}_{\mathrm{t}}$ is the interface transfer resistance, and CPE is the double-layer constant phase angle element.

The CPE element can be expressed by the following equation:

$$
Z_{C P E}=\frac{1}{Y_{0}^{-1}(j \omega)^{-n}}
$$

In the formula, ZCPE is the impedance of CPE, Y0 is the admittance of CPE, brown is the angular frequency of perturbation signal, and $\mathrm{n}$ is the dispersion index of CPE (depending on the roughness of the electrode surface and current distribution). When $\mathrm{n}$ is $0,0.5$, respectively, At 1 o'clock, CPE stands for pure resistance, warburg impedance, and pure capacitance.

At room temperature $\left(25^{\circ} \mathrm{C}\right)$, different mass concentrations of polyaspartic acid were added to the medium and the complex impedance planogram was obtained, as shown in Figure 4.

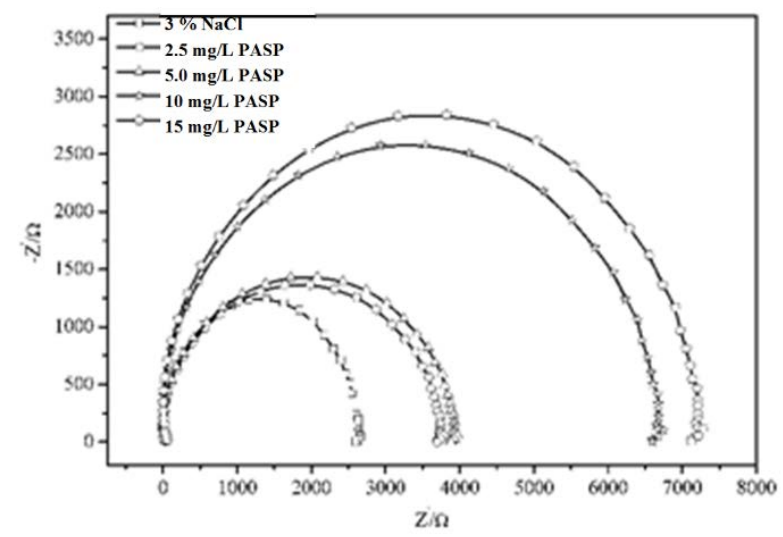

Fig.4 Nyquist plots for the copper in the different of PASP at $25{ }^{\circ} \mathrm{C}$

It can be seen from Figure 4 that the impedance spectrum shows different degrees of major semicircle characteristics. As the concentration of PASP increases, the semicircle increases and the Rt value of the electrode increases. Therefore, the corrosion rate decreases and the corrosion inhibition efficiency increases. 
Considering the analog circuit shown in Figure 4, fit the curve data in the graph and calculate the $\mathrm{R}_{\mathrm{t}}$ and $\eta$ values at $20{ }^{\circ} \mathrm{C}$. Then get another corrosion inhibition efficiency as shown in Table 2 . Among them:

$$
\eta=\frac{R_{t}-R_{0}}{R_{t}} \times 100 \%
$$

Where $\eta$ is the corrosion inhibition efficiency; Rt0 is the charge transfer resistance of the blank solution; Rt is the charge transfer resistance after the addition of the corrosion inhibitor.

Table 2 According to Nyquist workout resistance and the inhibitor efficiency

\begin{tabular}{|l|l|l|}
\hline $\mathrm{C}_{\text {PASP }}(\mathrm{mg} / \mathrm{L})$ & $\mathrm{Rt}(\Omega)$ & $\eta(\%)$ \\
\hline 0 & 2603.4 & - \\
\hline 2.5 & 3696.9 & 27.6 \\
\hline 5 & 3881.7 & 32.9 \\
\hline 10 & 6598.9 & 60.5 \\
\hline 15 & 7126.9 & 63.5 \\
\hline
\end{tabular}

As can be seen from Table 2, different concentrations of polyaspartic acid are added to the medium, and the resistance $\mathrm{R}_{\mathrm{t}}$ is transmitted as the concentration increases. As the value increases, the corrosion inhibition rate $\eta$ increases. The conclusions obtained have silitly difference with those obtained by the steady state polarization test but have similar trend.

\subsection{Adsorption Mechanism of PASP on Copper Electrodes}

Assuming there is no other process on the metal surface, only the adsorption and desorption process of the corrosion inhibitor, the adsorption coverage rate of the corrosion inhibitor on the metal surface when the adsorption and desorption processes reach equilibrium, and the concentration $\mathrm{C}$ of the corrosion inhibitor in the solution There is a balance relationship. This equilibrium relationship can be expressed by the adsorption isotherm. According to the experimental results, several isothermal equations of Frumkin, Temkin, Langmuir, and Freundlich were used to fit. The results show that the experimental results agree well with the Freundlich isothermal equation.

For example, the experimentally measured corrosion inhibition rate $\eta$ is used instead of the surface adsorption coverage $\theta$. According to the data shown in Table 2, we can get free energy of the adsorption during this process.

The corrosion potential data in Table 2 shows that with the addition of PASP, the corrosion potential Ecorr does not change significantly, and it can be inferred that the corrosion mechanism is a "covering effect" and the corrosion rate is turbid, indicating its coverage. Assuming PASP is on the copper surface ans adsorption conforms to Langmuir adsorption isotherm

$$
\frac{C}{\theta}=\mathrm{C}+\frac{1}{B}
$$

Where $\mathrm{B}$ is the Langmuir constant and $\mathrm{c}$ is the inhibitor concentration:

Figure 5 is obtained by plotting $\frac{C}{\theta}$ versus c, and the slope and intercept are obtained by fitting the line. Then we get the following relationship:

$$
\frac{C}{\theta}=6.12 \mathrm{C}+1.14
$$

The fitting $\mathrm{R}=0.95$ indicates that the adsorption of PASP on the copper surface is in good agreement with Langmuir isotherm adsorption. Substituting the fitting data into equation (3) yields B $=100 \mathrm{~L} \cdot \mathrm{mol}^{-1}$ (The standard of B should be $\mathrm{L} \cdot \mathrm{mol}^{-1}$, and the amount of aspartic acid per monomer in polyaspartic acid is $114 \mathrm{~g} \cdot \mathrm{mol}^{-1}$, so $1 / \mathrm{B}=1.14 / 114$, that is, $\mathrm{B}=100 \mathrm{~L} \cdot \mathrm{mol}^{-1}$ ). The relation between $\mathrm{B}$ and the adsorption Gibbs free energy $\Delta \mathrm{G}_{0}$ is: $\mathrm{B}=\frac{\mathrm{e}^{-\Delta \mathrm{G}_{\mathrm{m}} / 2.3 \mathrm{RT}}}{\mathrm{C}^{*}}$

Where $C^{*}=55.5$ is the solubility of water in solution in mol/L. Then calculated by equation, $\Delta \mathrm{G}_{0}$ 
$=-49.13 \mathrm{~kJ} / \mathrm{mol}$.

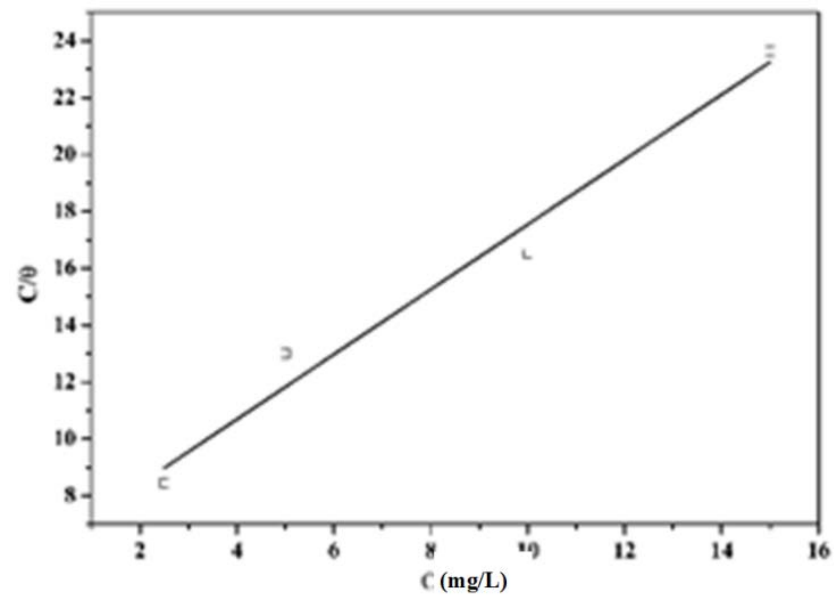

Fig.5 Langmuir isothermmodel of PASP on copper

According to Brdika's research results, the standard adsorption free energy of chemisorption and physisorption is limited to about $100 \mathrm{~kJ} / \mathrm{mol}$, which greater than this value is chemical adsorption, and less than this value is physical adsorption. This article yielded $-49.13 \mathrm{~kJ} / \mathrm{mol}$, indicating PASP. The inhibition effect on the copper surface may be due to chemical adsorption.

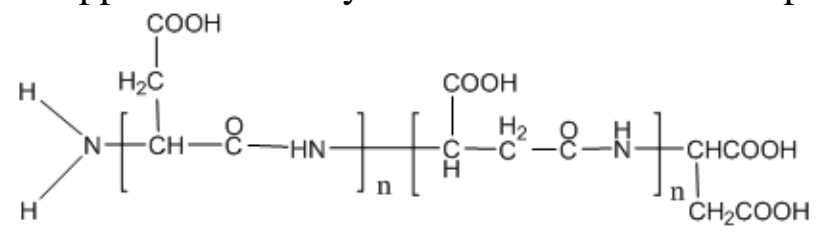

Fig.6 Molecular structure of PASP

From the perspective of the molecular structure of PASP in Figure 6, it is a water-soluble macromolecular polypeptide chain, with polar groups such as carboxyl, carbonyl, amino and nitrogen hydrogen bonds. Oxygen and nitrogen atoms in the carbonyl group contain lone pair electrons. Oxygen atoms also contain $\pi$ bonds, which can both chemically adsorb with copper atoms, align polar groups on the metal surface, and increase the anodic reaction resistance; non-polar groups have a large steric hindrance, hindering the cathode oxygen Copper surface diffusion inhibits the reduction of the cathode. Therefore, it is a physical combine with chemical adsorption process.

\section{Conclusions}

The following conclusions were obtained from electrochemical experiments:

(1) In simulated sea water systems, PASP has a corrosion-inhibiting effect on carbon steel, which is a corrosion inhibitor that mainly suppresses the anode. With the increase of the concentration of PASP, the corrosion inhibition efficiency increases; when the concentration reaches $15 \mathrm{mg} / \mathrm{L}$, corrosion inhibition efficiency can reaches $77.2 \%$.

(2) In the experimental temperature range, the adsorption of PASP on the copper surface follows the Langmuir adsorption isotherm; at $25^{\circ} \mathrm{C}, \mathrm{B}=100, \Delta \mathrm{G}_{0}=-49.13 \mathrm{~kJ} / \mathrm{mol}$.

\section{References}

[1] Xu Q, Zhu L, Cao W et al. Inhibition Action and Adsorption Behavior of Environment-Friendly Inhibitor Poly-Aspartate on Copper. Acta Physico-Chimica Sinica, 24(9), 1724-1278 (2008).

[2] Zhou X, Fan H. Corrosion Inhibition Behavior of Copper and Carbon Steel by Polyaspartic Acid. Materials Protection, 38(3), 59-61 (2005).

[3] Xu Q, Shan Z, Zhou Gl. Corrosion inhibition of compounded PASP for cupronickel B30 in 30\% $\mathrm{NaCl}$ solution. East China Electric Power, 34(5), 16-19 (2009). 
[4] Yang W, Gooding J, Hibbert D. Redox voltammetry of sub-parts per billion levels of Cu2+ at polyaspartate-modified gold electrodes. Analyst, 126(9), 1573-1577 (2001).

[5] Zhou G, Yin R, Wan Z et al. Electrochemical and photoelectrochemical study of the self-assembled monolayer phytic acid on cupronickel B30. Corrosion Science, 50(12), 35273533 (2008).

[6] Xu Q, Ding S, Yun Hu et al. Inhibition Action and Adsorption Behavior of PESA on Copper. Advanced Materials Research, 148-149 (2010).

[7] Rao B, Meddy M. Formation, characterization and corrosion protection efficiency of selfassembled 1-octadecyl-1H-imidazole films on copper for corrosion protection. Arabian Journal of Chemistry, 3, (2008).

[8] Deng X, Xu Q, Yun H et al. Progress in Research on Corrosion Performance of Copper and Copper Alloys with Super-hydrophobic Surface. Corrosion \& Protection, 1(01), (2012)

[9] Ma Q, Zhang X, Han L. Synthesis Research of Corrosion Inhibitor Similar to Polyepoxysuccinic Acid for Carbon Steel in Sea Water. Advanced Materials Research, 291(294), 192-196 (2011).

[10] Zhang J, Li G, Zhu Yl. Corrosion Inhibition Performance of Polyepoxysuccinic Acid and its Complex. Acta Physico-Chimica Sinica Corrosion Science \& Protection Technology, 21(2), 128130 (2010). 